\title{
THE APPLICATION OF 3D LASER SCANNING IN THE PROTECTION OF GROTTOES AND CARVINGS IN CHINA
}

\author{
Li Yumin $^{\text {a }}$, Zhang Rong ${ }^{\text {a }}$, Zheng Yu ${ }^{\text {a }}$ \\ ${ }^{a}$ Beijing Tsinghua Urban Planning \&Design Institute, Cultural Heritage Conservation Centre, Beijing, P.R.China
}

KEY WORDS: 3D laser scanning, grottoes and carvings, protection, demonstration, interpretation

\begin{abstract}
:
With the development of 3D laser scanning technology, it has been widely used in the recording and conservation of stone cultural relics. A lot of grottoes and carvings in national key cultural heritage protection units in Shanxi, Gansu and Sichuan areas of China have been scanned by 3D laser scanner in their protection projects. In this paper, via these 3D laser scanning projects of the grottoes and carvings, technical essentials of indoor and field work in the 3D laser scanning technology of stone cultural relics are summarized. At the same time, different devices were selected and different device parameters were set on the basis of characteristics of specific relics. Thus the current situation of the relic was recorded precisely with the least storage space and the shortest working time. At last, digital results including 3D models, 2D drawings, the videos for exhibition and etc with powerful operability which meet different needs are obtained based on the original point cloud. This paper can provide some beneficial reference and experience for recording and conservation of the grottoes and carvings relics in the future.
\end{abstract}

\section{THE CURRENT SITUATION OF STONE CULTURAL RELICS IN CHINA}

\subsection{Introduction}

As the eastward of Buddhism, the cutting of grottoes have risen all over China, especially in the the Yangtze river basin, Yellow river basin, the silk road and so on. According to statistics, just the number of stone cave temple and the national key cultural relics' protection units of grotto has reached to 286 . The cutting of grottoes began from Han dynasty, was prevalent in Northern Wei, Sui and Dang dynasties, song dynasty and continued to Ming and Qing dynasties. The grottoes are rich in content, such as buildings, statues, murals, relief and so on. These relics are Valuable Information for research on social economy, politics, religion, art and architectural engineering of China ${ }^{[1]}$.

\subsection{The problem of grottoes' current situation}

Through a long time, much of stone cultural relics were badly damaged. The main reason include following aspects:

1. Erosion of natural forcing

Rainfall and groundwater gave rise to water seepage and damp, Thus Caused microbial disease and Surface suffused with salt, even caused missing of detail feature and dropping of colour. On the other hand, geological disaster might give rise to damage of statues and wall paintings.

2. Change of the environment

As urban construction and development of modern industry, cultural relics in the grotto were affected by the environment. For instance, Acidic floating dust, floating dust and the change of environmental humidity could accelerate the weathering of grottoes statues.

3. Man-made sabotage

From the 19th century to the 20th century, lots of grotto relics had been lost overseas. In addition, many grotto relics were stolen by criminals in recent years.
Because a complete recording is the most fundamental and reliable basis for the work of protecting cultural relics, a fundamental recording for current situation of grotto relics is necessary before all the problems are solved.

\subsection{Recording method for current situation of Grotto Relics}

The investigation on grotto relics in China has begun in the $1930 \mathrm{~s}^{[2]}$. The original surveying method for grottoes depended on the manual surveying. The advantage of this method is simple and cheap, but it is time-consuming and has low efficiency.

As the development of the technology on surveying and compute, close range photogrammetric is more and more used in surveying of Grotto Relics. This can make up the difference of manual surveying and improve the surveying precision and efficiency, however, because of the lack of expression, this method can cause a loss of detail information.

In recent years, a new type of recording technology for three-dimension information-3D laser scanning is introduced to protection work for grotto relics. 3D laser scanning system is measuring instrument for three-dimension coordinate with many kinds of high-tech. It adopts non-contact and active measuring method, can acquire high-precision three-dimension data and can scan any type of object. At the same time, it is not restricted by light and can quickly acquire the surface information. 3D laser scanning has a wide application prospect in protection work for grotto relic ${ }^{[3]}$.

\section{THE THEORY AND PARAMETER SETTING OF 3D LASER SCANNING}

\subsection{The theory of 3D laser scanning}

According to the location principle of 3D laser scanning, as shown in Figure 1, Origin of coordinates is located in the centre 
of the instrument. $\mathrm{Y}$ axis is along the direction of scanning during initialization of the instrument. $\mathrm{Z}$ axis is just sticking straight up. The direction of $\mathrm{X}$ axis can be settled by right-hand rule. By doing this, the three-dimension coordinate of scanning point can be acquired.

Where:

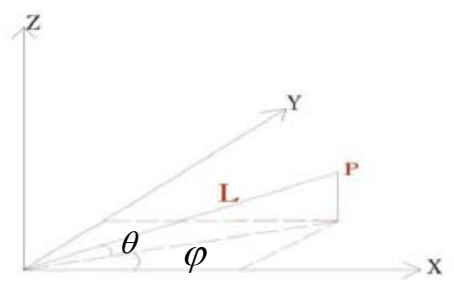

Fig. 1

$$
\left\{\begin{array}{l}
X=L \cos \theta \cos \varphi \\
Y=L \cos \theta \sin \varphi \\
Z=L \sin \theta
\end{array}\right.
$$

Where $\mathrm{L}$ denotes the distance of scanning, $\theta$ represents the vertical angle. Because of the error propagation, the precision of scanning point can be described as follow ${ }^{[5]}$ :

$$
\sigma_{P}^{2}=\sigma_{X}^{2}+\sigma_{Y}^{2}+\sigma_{Z}^{2}=\sigma_{L}^{2}+L^{2} \sigma_{\theta}^{2}+L^{2} \sigma_{\varphi}^{2} \cos ^{2} \theta
$$

Where $\sigma_{P}^{2}$ denotes the precision of scanning point, $\sigma_{L}^{2}$ represents the precision of ranging accuracy, $\sigma_{\theta}^{2}$ is the precision of angular accuracy. It can be seen from the equation (2) that the precision of scanning point is determined by the precision of ranging accuracy, the precision of angular accuracy, the distance of scanning and the vertical angle. Among them, $\sigma_{L}^{2}$ and $\sigma_{\theta}^{2}$ determines the accidental error, $L$ and $\theta$ determines the systemic error.

In general, the smaller the $L$ and the bigger the $\theta$ provide higher scanning precision. In practical application, the bigger $\theta$ and smaller $L$ should be chosen to ensure enough scanning precision, if possible.

\subsection{Setting of scanning-point space}

Except the distance of scanning and vertical angle which are set correctly, point cloud density is a critical indicator to ensure that the point cloud data which is acquired by $3 \mathrm{D}$ laser scanning can describe the grotto statuary precisely. As shown in Figure.2. Sparse point spacing cannot describe the characteristic of Complex surface accurately, however, if choosing the minimum distance of scanning which the 3D laser scanning instrument can supply, it will make it hard to save, display and edit the data How to set the distance of scanning will depend on the minsize of the characteristic. It is possible to describe the grotto statuary precisely only by choosing small enough distance of scanning. Assume that Accuracy of the characteristic described by point cloud data is $\mathrm{Q}, \mathrm{m}$ denotes the minsize of the characteristic, $\lambda$ represents the distance of scanning. Then their relationship can be described as follow:

$$
\mathrm{Q}=(1-\lambda / \mathrm{m})
$$

Assume the minsize of the characteristic is $5 \mathrm{~mm}$. As shown in Table1.

\begin{tabular}{|c|c|c|}
\hline$\lambda(\mathrm{mm})$ & $\mathrm{Q}$ & status \\
\hline 10 & -1 & error \\
\hline 5 & 0 & error \\
\hline 2.5 & 0.5 & correct \\
\hline 1 & 0.8 & correct \\
\hline
\end{tabular}

When $\mathrm{Q}$ is close to 1 , the Accuracy is higher ${ }^{[4]}$

After considering working time and data size together, finding out the minsize of the characteristic, $\lambda$ can be settled at last.
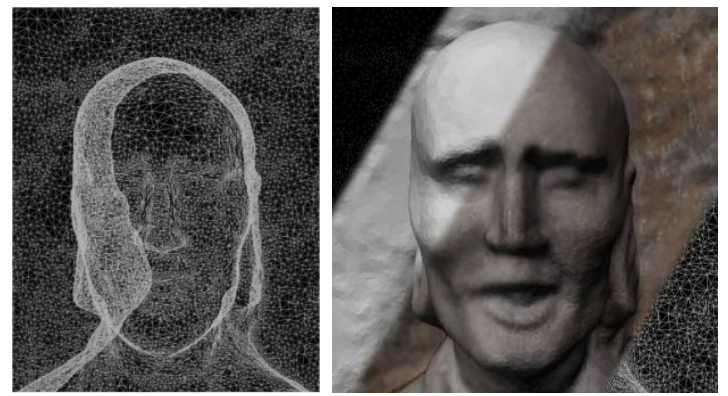

Fig.2 different point space-different character

In a word, only by setting correct parameters can be the satisfactory data acquired. To provide Fundamental Date for Grottoes cultural relics, digital library system of Grottoes cultural relics can be established by combining the threedimension data with repairing files and construction record which acquired by investigation.

\section{APPLICATION OF 3D LASER SCANNING ON THE CONVERSATION OF GROTTOES AND CARVINGS}

Relics can't be substitute because of its authenticity and integrity. We can't get the point in the protecting projects without knowing them. So the most important problems we should solve in culture relics protection are making up for the lack of integrity and authenticity of cultural relics as far as possible, and setting the appropriate protection of cultural relics. Protection planning formulation mainly contents three stages, which are current situation investigation, analysis and evaluation, measures formulated. 3D laser scanning provides accurate data source and new methods throughout each stage, in order to protect the authenticity and integrity of the cultural relics.

\subsection{The assessment and research of grottoes current situation}

It is critical to detailed investigate the disease of the cultural relics in the grotto, find the distribution of the disease, estimate the degree of harmfulness for formulating conservation plan.

The orthoimage is one main way of manifestation of grottoes current situation. It is very simple to acquire the orthoimage accurately of the cultural relics in the grotto with the surface model which is got by $3 \mathrm{D}$ laser scanning. It can be seen from the Figure3. According to the triangular grid model, the orthoimage can be acquired directly.

In addition, because the cultural relics in the Grotto and occurrence of rock mass are irregular solid, the error is big by 
the way of the statistical disease in the plane. To reduce the statistical error, the disease information can be defined directly for triangle patch on the model and the area of disease can be measured conveniently by the way of triangular grid model. In the plan of protection for thousand-buddha cliff in Guangyuan, as shown in Figure 3, the statistical area of disease can be acquired exactly and the distribution of disease can be displayed directly by $3 \mathrm{D}$ laser scanning and defining the type of disease on every triangle patch.

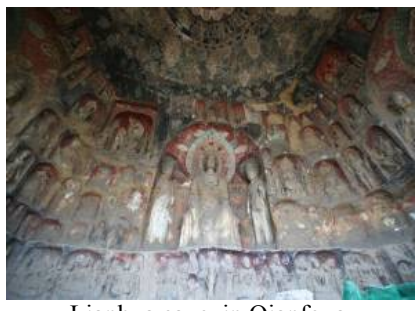

Lianhua cave in Qianfoya

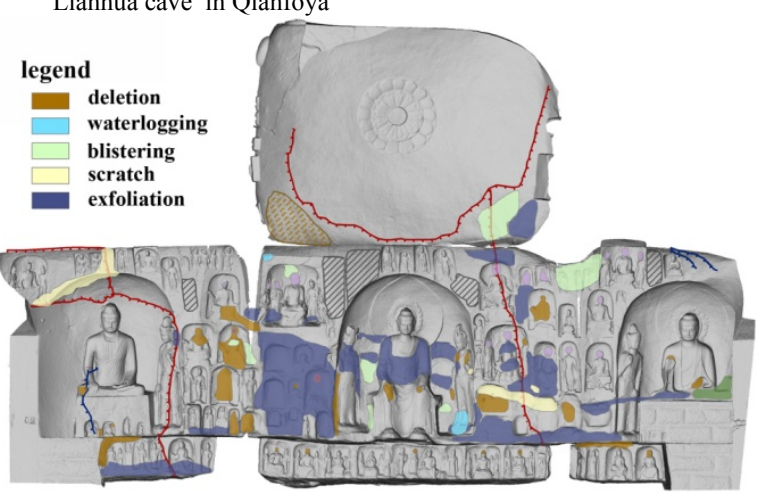

Fig.3 Distribution of disease in lianhua cave

\subsection{Conservation measures taking}

Sometimes relics need to be reinforced in the structures, because of the instability of the rock mass. It is easy to find the direction, the angle and the distance of inclination of the relics. Furthermore, components falling from the relics can also be precise in the system. It provides a new effective method for relic's protection. For example, the Jingmu tower in Sichuan province which was built in Song dynasty was badly damaged in the earthquake. As shown in Figure4. It leaned to the southwest more seriously and obviously. The stone component fell down from the top of the tower, at the same time breaking down the side of components of the tower ${ }^{[6]}$. While simply having access to a clear and accurate record of 3D model with 3D laser scanning of the tower helps the planner to determine the appropriate action for its protection. And it can also bring the fallen components back virtually which have fallen down in the system. As shown in Figure5 and Figure6.

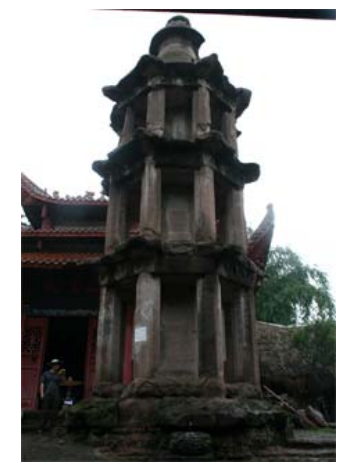

Fig.4 Jingmu Tower in Sichuan

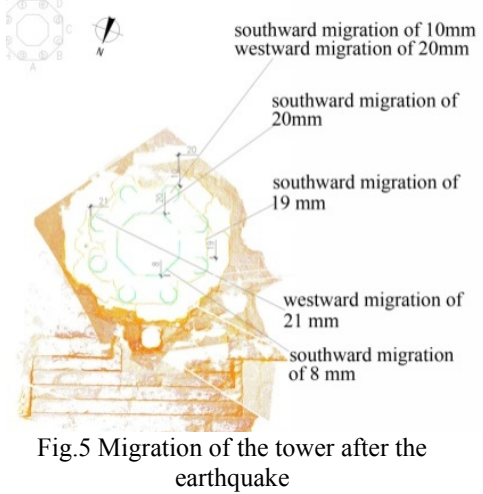
earthquake
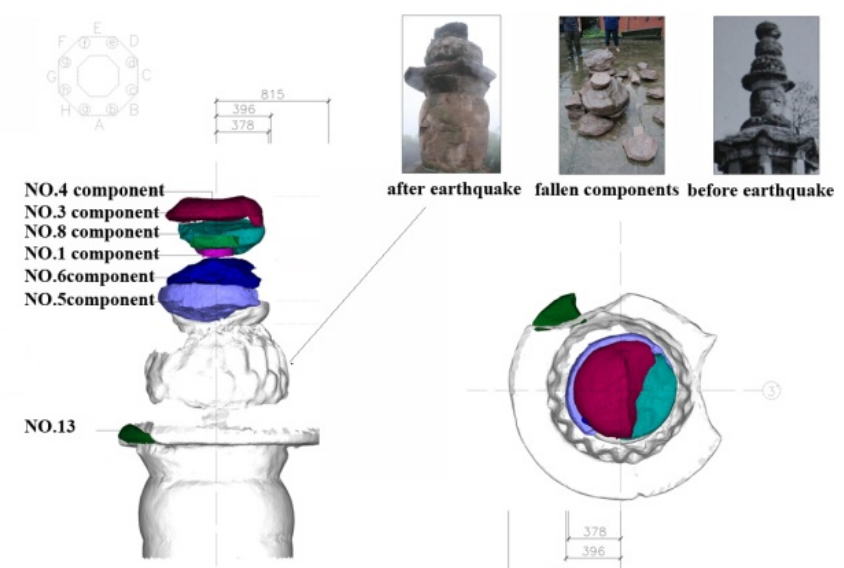

Fig.6 The sketch of the restored jade

\subsection{Demonstration and Interpretation}

The data which is acquired by $3 \mathrm{D}$ laser scanning contains rich spatial information. It helps to provide many new ideas for the exhibition of cultural relics.

Because of the construction of reservoir, most of statues and murals of Tiantishan grottos which is built during Northern Liang was relocated to somewhere else. The authenticity and integrity of Tiantishan grottoes were destroyed. As shown in Figure7 and Figure8. At present, the cultural connotation, historical information and value contained in these statues and murals either displayed in museum or in cavern of north shore of the reservoir cannot be interpreted and delivered well. As shown in Figure9. This manner can only be called preserving instead of protection. The research of virtual restoration was preceded by means of historical photos, grottoes shape data and 3D laser scanning for these cultural relic. Because the color digital model of statues and murals was virtual restored according to the grottoes shape and the relationship of every part is established, the historical information can be experienced by the public completely and truly. As shown in Figure 10.

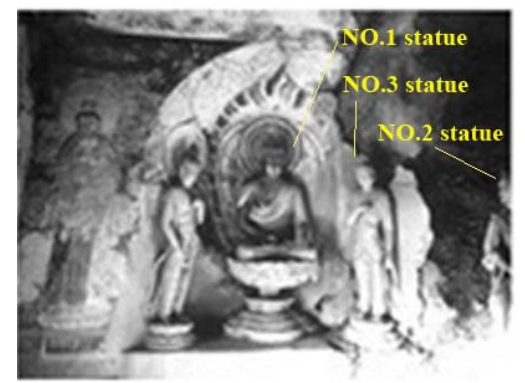

Fig.7 NO.2 cave of Tianlongshan in 1922

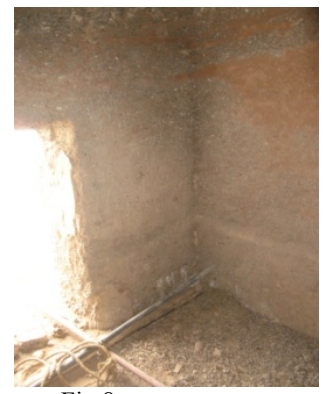

Fig. 8 current cave

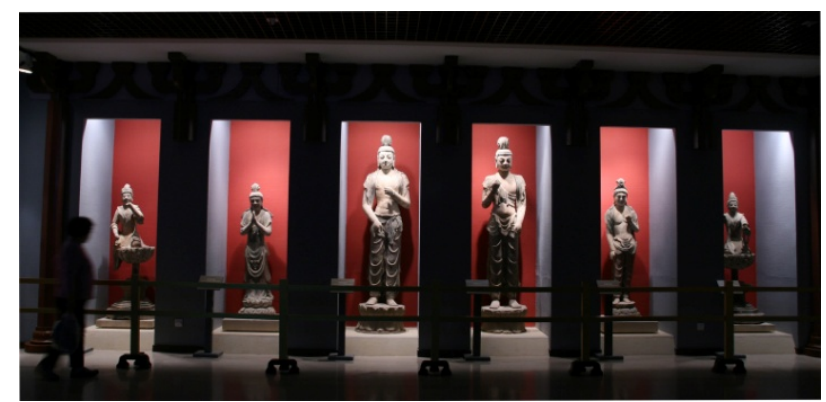

Fig.9 the exhibition of statues from Tiantishan Cave 


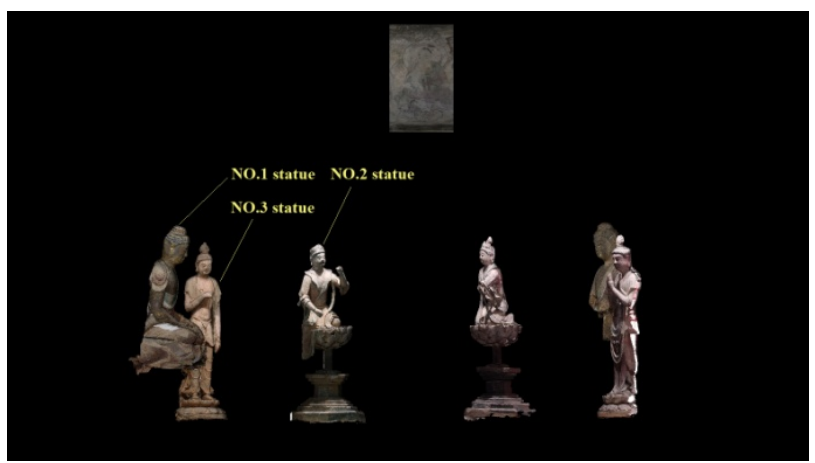

Fig.10 The restored setup of no.2 cave virtually

\subsection{Digital reconstruction of loosed relics}

There are so many relics loosed from China to other countries all over the world, and it's difficult to recover them. In this case, scanning of those frescoes and grottoes, and putting them together digitally in the $3 \mathrm{~d}$ virtual system become more and more important. Take the Tianlongshan Cave in Shanxi province as an example. The cave was dogged in Eastern Wei dynasty. Unfortunately, almost all the heads of statues and exquisite frescos were stolen during the 1920 s to 1930 s. The authenticity and integrity of the relic were greatly damaged. As shown in Figure11 and Figure12. And fortunately, 61 percent of the looted relics of the Tianlongshan Cave can be found where they are. At present, the caves and those incomplete statues had been scanned accurately. As shown in Figure13. If circumstances allowed, the 3D models of those looted relics can be obtained by 3D laser scanning, and thus the statues and the whole cave can be renovated virtually. As shown in Figure14. These digital models which can be shared among international relevant organizations will bring many advantages. On the one hand, the historical value, artistic value and social value in the relics can be displayed comprehensively; on the other hand, it is a positive measure for bringing Chinese artifacts back to China.

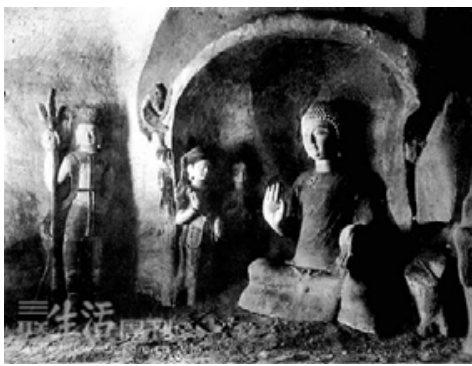

Fig.11 Grotto of Tianlongshan in 1922

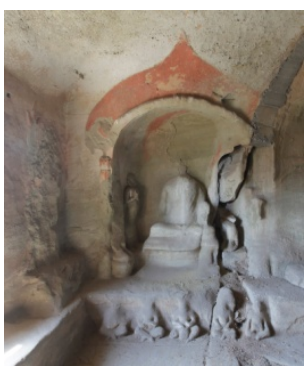

Fig.12 current grotto

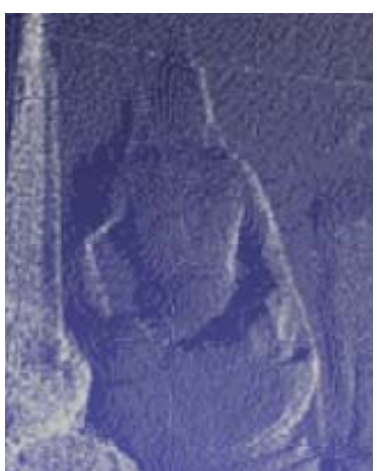

Fig.13 The 3D point cloud and surface model
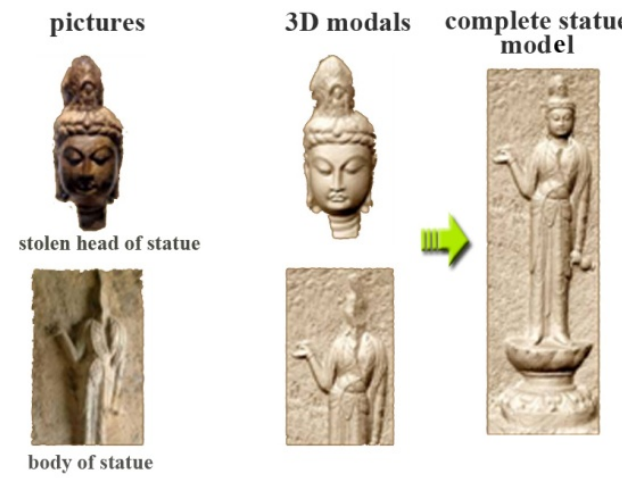

Fig.14 Digital reconstruction of head and body of a statue

In a word, the content displayed and ways of interpretation are no longer two-dimensional pictures and documents, it becomes more lively and tridimensional for presentation in visitor centres, museums, through the internet and the media (enhancing accessibility and helping to improve understanding) by 3D laser scanning.

\section{CONCLUTION}

3D laser scanning has played an important part in conservation plan of the stone caves and grottoes at any stage of a project. Tasks that might be considered as suitable for application of laser scanning could include any of the following:

1. 3D laser scanning may provide a detailed record of the relic through non-contact surveying. Furthermore, the scanner can obtain what we can see in this direction, even the parts which are easy to miss by other survey ways. And the work efficiency can also be improved greatly by the way of high-speed scanning. 2. Simply having access to a clear and accurate record of 3D model with 3D laser scanning provides necessary analyze data for assessment, and it also helps the planner to determine the appropriate action for protection of the stone caves and grottoes in the three-dimensional space.

3. 3D laser scanning might provide abundant exhibition content, and bring more distinctive interpretation of the concept. Scanning may improve the accessibility of the stone relic, to aid tourists and experts understanding, or improve engagement with the general public.

However, it's lack of specificity in the process of application of the conservation project. Firstly, feature identification and extraction of relics is partly depended on manual work. Secondly, it is still very difficult to load and edit massive data. Thirdly, the 3D data products are so diverse and not compatible enough with the regular software in plan and design. And because of the sheer cost, it is difficult to use this technology in relics protection widely.

As the development of computer and its related technology, the technology of 3D laser scanning can be further refined. Combined with the technology of remote sensing, GIS and virtual reality, rich three-dimensional information can be further sought to meet the more needs of cultural relics preservation.

\section{REFERENCES FROM JOURNAL}

[1] Huang KZ. Conservation Status of Grottos in China [J]. DunHuang Research, 1994(1). pp.18-23. 
[2] Liu YQ. Discussing on the Work of Grotto Archaeology Surveying and Mapping [J]. DunHuang Research, 1996(1), pp. $158-160$

[3] Zhang RJ, Wang YM. Research on 3D Reconstruction Using Laser Scanning Data Acquired from Ancient Architecture[C]. Science of Surveying and Mapping, Beijing, China, Vol132 No.2, Mar. 2007, pp. 269-273.

[4] 3D Laser Scanning for Heritage, English Heritage, 2011

[5] Ding W. Terrestrial laser scanning technology and error analysis of point cloud[J]. Geotechnical Investigation \& Surveying, 2009(2), pp.447-452.

[6] Zhang R, Li ZE, Xu SC. Rescue Preservation of the Peacock Cavity Canon tower in Anyue Grottoes after the 5.12 WenChuan Earthquake: Application of three- dimension laser scan technology and computer simulation technology to its conservation[J]. SCIENCES OF CONSERVATION AND ARCHAEOLOGY, 2010(22):5, pp.40-47. 